\title{
Osteonecrosis of jaw beyond antiresorptive (bone- targeted) agents: new horizons in oncology
}

\section{Vittorio Fusco, Daniele Santini, Grazia Armento, Giuseppe Tonini \& Giuseppina Campisi}

To cite this article: Vittorio Fusco, Daniele Santini, Grazia Armento, Giuseppe Tonini \& Giuseppina Campisi (2016) Osteonecrosis of jaw beyond antiresorptive (bone-targeted) agents: new horizons in oncology, Expert Opinion on Drug Safety, 15:7, 925-935, DOI: 10.1080/14740338.2016.1177021

To link to this article: http://dx.doi.org/10.1080/14740338.2016.1177021

Apr 2016.

Published online: 03 May 2016.

Submit your article to this journal $\pi$

Џ Article views: 132

View related articles $\smile$

View Crossmark data $\asymp$ 


\title{
Osteonecrosis of jaw beyond antiresorptive (bone-targeted) agents: new horizons in oncology
}

\author{
Vittorio Fusco ${ }^{a}$, Daniele Santini ${ }^{b}$, Grazia Armento ${ }^{b}$, Giuseppe Tonini ${ }^{b}$ and Giuseppina Campisic

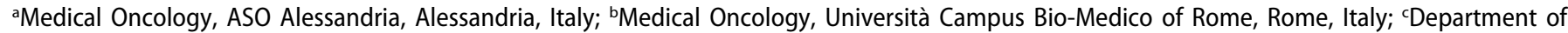 \\ Surgical, Oncology and Dental disciplines, Università degli Studi di Palermo, Palermo, Italy
}

\begin{abstract}
Introduction: Osteonecrosis of the jaw (ONJ) is a clinically important, potentially painful and debilitating condition, which can affect the quality of life of cancer patients. Since 2003, ONJ appeared as a Bisphosphonate(BP)-related class effect, and the term Bisphosphonate-Related Osteonecrosis of the Jaw (BRONJ) was widespread.

Areas Covered: Under discussion in this review is the fact that ONJ cases have been reported after treatment including antiangiogenic agents and other "targeted therapy", with and without BPs. Consequently, the comprehensive term Medication-Related Osteonecrosis of the Jaw (MRONJ) has been introduced. The clinical aspects and the prognosis of ONJ associated with these new drugs are still less reported, but basing on their pharmacodynamics, they could be different from the well-known BRONJ. Accordingly, recommendations largely in use for BRONJ should be extended to these new forms, but critically applied and with respect to the individual risk assessment.

Expert Opinion: There is a high risk of underdiagnoses for ONJ due to a lack of awareness, and too much restrictive or incomplete diagnostic criteria; at the same time, with regard to ONJ associated to the new non -antiresorptive agents, described here, we observe the strong need to improve the defining of any distinguished feature in their diagnosis, prevention and therapy.
\end{abstract}

ARTICLE HISTORY

Received 10 January 2016

Accepted 6 April 2016

Published online

29 April 2016

\section{KEYWORDS}

Osteonecrosis of the jaw (ONJ); Bisphosphonate; Bisphosphonate-Related Osteonecrosis of the Jaw (BRONJ); denosumab;

Medication-Related Osteonecrosis of the Jaw (MRONJ); bevacizumab; aflibercept; sunitinib; temsirolimus; everolimus

\section{Introduction}

Osteonecrosis of the jaw (ONJ) represents a clinically important, potentially painful, and debilitating condition that can meaningfully affect the quality of life (QoL) of cancer patients. Since 2003, ONJ appeared as a bisphosphonate (BP)-related class effect, and the term bisphosphonate-related osteonecrosis of jaw (BRONJ) was used. Recent studies have shown that the new receptor activator of nuclear factor kappa-B ligand (RANKL) inhibitor denosumab can equally induce ONJ. Furthermore, ONJ cases have been reported after treatment including antiangiogenic agents and other 'targeted therapy', with and without BPs, in cancer patients. Consequently, the term medication-related osteonecrosis of jaw (MRONJ) has been recently introduced in medical literature.

As every year novel drugs with antiangiogenic properties and multi-target mechanisms are introduced in the anticancer therapeutic armamentarium, clinicians and patients have to give high attention to oral health problems during treatment with new agents, in order to distinguish between stomatitis, a very common side effect of most of these drugs, and potential signs of early MRONJ, with or without bone exposure.

Risk factors related to ONJ are known, but it does not exist as a complete picture. Radiotherapy of head and neck tumors, periodontal diseases, jawbone surgery, and edentulous zones or prosthesis have been reported as related with the development of ONJ.[1] Among the risk factors, it is also considered - the systemic treatments, especially if prolonged, with BPs.[2] However, the concomitant dentoalveolar surgery and periodontal/dental diseases are the main risk factors.

Oral surgery (implantology and periapical surgery) increases the risk of ONJ, as well as concomitant imbalance of oral health (e.g. dental inflammation and dental abscesses). Finally, there are risks associated with tumor histology, age, and race. Multiple myeloma was most often associated with ONJ compared to other solid tumors [3] in first reports. Moreover, it is unknown what impact these drugs have on patients' oral-QoL, specifically in relation to any subclinical manifestations that may not be routinely identified during cancer therapy. Although it is not known whether the injuries caused by osteoclasts inhibiting agents are equal to those caused by antiangiogenetic agents, literature shows how the QoL is adversely affected by osteomyelitis of the jaw during BPs or denosumab treatment. The first empirical evidence that BP-associated ONJ significantly impairs QoL, with an increasing effect with stage severity was published in 2011.[4] ONJ adversely affects a wide range of oral health, and the magnitude of the negative QoL effects of ONJ is equivalent to other cancer treatment side effects that influence treatment decisions.[5,6] By translating evidences emerging in studies of inhibitors of osteoclast activity, it could easily figure out how the QoL of patients with ONJ may be compromised by other agents, such as anti-vascular endothelial growth factor (VEGF), 


\section{Article highlights}

- Osteonecrosis of the jaw (ONJ) cases have been reported in cancer patients after treatment including antiangiogenic agents and other 'targeted therapy', with and without antiresorptive drugs (bisphosphonates, denosumab).

- New patient populations are at risk of ONJ.

- The number of ONJ cases in patients treated with innovative anticancer drugs are increasing.

- There is a high risk of underestimation due to a lack of awareness, and restrictive diagnostic criteria for ONJ, especially if a frank bone exposure is missing.

- Observational study and experimental research are needed to define any distinguished feature in the diagnosis, imaging, prevention and therapy of targeted-therapy associated ONJ.

This box summarizes key points contained in the article.

mammalian target of rapamycin (mTOR) inhibitors, and tyrosine kinase inhibitors (TKIs). VEGF is essential for bone repair mechanisms so the use of anti-VEGF drugs (both monoclonal antibodies and TKIs) could theoretically cause ONJ, even without concomitant use of BPs.

Even if rarely, a delayed or missed diagnosis of ONJ might exist in life-threatening or lethal disease (necrotizing fasciitis or neck cellulitis) [7-10]: so, every effort to avoid delayed or missed diagnosis is warranted.

The first case report associating bevacizumab with the onset of ONJ was published in 2008 [11]; subsequently, it was retrospectively analyzed for the data of safety on the use of bevacizumab in improving the clinical outcome of patients with breast cancer (ATHENA, AVADO, and RIBBON-1) [12] demonstrating that the concomitant use of bevacizumab plus intravenous BPs significantly increased the risk of ONJ compared with the use of bevacizumab or BP alone. The same mechanism that underlies the hypothetical pathogenesis of ONJ with the use of bevacizumab may be shifted to the administration of TKls in particular with sunitinib in metastatic renal carcinoma and gastro intestinal stromal tumor (GIST). Wide use of sunitinib in clinical practice, especially in patients with localization of bone disease that require agents inhibiting osteoclast activity, led to the identification of patients who reported ONJ disease sites.[13] The population of patients with renal cancer was also analyzed for the use of inhibitors of mTOR, such as everolimus and temsirolimus. In fact, there were two published case reports $[14,15]$ in which, patients previously treated with BPs have reported ONJ. Further studies are needed to determine what is the role of the individual drugs, if there is a synergy or a trigger component. A diagnostic algorithm designed on the emerging evidence is necessary in order to identify high-risk patients, for which a preventive and coded approach is necessary. The proper stratification of patients' risk class could help in improving the decision-making and improving the patient's adherence to the treatments with a hypothetical consequent betterment of clinical outcome.

\section{Definition of ONJ}

The definition of ONJ is controversial, with evident consequences on clinical practice, trials and epidemiological studies. The most applied definition of ONJ is the one that was originally proposed by a Task Force of the American Association of Maxillofacial Surgeons (AAOMS) in 2006 and published in 2007,[16] based on presence of bone exposure lasting at least 8 weeks in patients treated with BPs and never treated with radiation on head and neck region. That definition was linked to a staging system based on the presence of clinical signs and symptoms, and it was also substantially approved by a Task Force of the American Society of Bone and Mineral Research (ASBMR).[17] In succeeding years, the term BRONJ entered largely in use in medical literature, also when the first cases of ONJ after treatment including antiangiogenic agents and denosumab began to be reported. $[18,19]$ In the same years, clinical practice and literature $[20,21]$ showed increasing evidence of symptomatic cases of BP-related jawbone alterations without frank bone exposure, therefore, questioning the ONJ definition.

In 2009, the AAOMS Task Force [22] did not modify the definition but added a 'stage 0 ' to classify cases with signs and symptoms of the jaws without bone exposure; the contradictory nature of that position paper was underlined by several authors and researchers calling for a new larger definition to include the non-exposed ONJ.[23-30] Finally, in 2014, an AAOMS special committee released a third position paper [31] changing the term from BRONJ to medicationrelated ONJ (MRONJ) in order to include cases linked to the treatment with denosumab and those arising after antiangiogenic drugs and targeted therapies; the document enlarged the definition of disease to include cases with 'bone that can be probed through an intraoral or extraoral fistula in the maxillofacial region that has persisted for longer than 8 weeks' and confirmed the existence of a 'stage 0 category' for patients with signs and symptoms without bone exposure. On the other hand, an international task force recently confirms the ASBMR 2007 definition without the AAOMS 2009 and 2014 amends.[32,33] These controversies on definition are clearly of paramount importance as a possible cause of incorrect estimation of ONJ incidence on clinical studies, as well as patient selection and follow-up duration, [34] and they might also induce under-diagnosing of ONJ cases in clinical practice. Furthermore, the purely clinical-based definition of ONJ is questioned by the emergent role of imaging studies.[35,36]

\section{ONJ related to drugs other than antiresorptives}

Between 2003 and 2008, the medical literature registered an explosion of Reports and Papers regarding ONJ related to BPs, the so-called BRONJ.[37] The etiology of BRONJ has not yet been completely elucidated [38,39]; it is probably of multifactorial origin, due to different possible mechanisms:

- osteoclast inhibition, consequent bone resorption arrest, and turnover disruption;

- bone infection;

- the antiangiogenic effect of various BPs (such as zoledronic acid);

- immune system modification; 
- a BP-related toxic effect on cells other than osteoclasts (i. e. soft tissue cells); and

- other possible co-factors, such as microtrauma and vitamin $D$ deficiency.

The number of biological and animal model data is growing, but currently there is no single phenomenon explaining the variety of ONJ, which has arisen after intravenous or oral BP treatment.[40] After bone remodeling alterations, the antiangiogenic activity of BPs has been suggested as one of the main determinants of the onset of ONJ $[41,42]$; the impact of VEGFR genetic polymorphism has also been investigated.[43] Further data have been obtained from studying the apoptosis of circulating endothelial cells following the treatment with BPs [44] and from several animal models. The role of tooth extraction or other alveolar trauma in the onset of ONJ is not entirely known although it may well be a possible trigger as well as a sentinel of pre-existing, underlying bone disease. The risk factors for BRONJ, classified as systemic or local, have been largely described.[40] Denosumab is a completely humanized monoclonal antibody, an inhibitor of RANKL (which is secreted by osteoblasts), acting as a bone turnover blocker. Consequently, and theoretically, denosumab-related ONJ disease could have characteristics unlike those of BRONJ. Further large-scale studies are required to ascertain whether denosumab-related ONJ cases do have a more favorable outcome after 'conservative' therapies (antibiotics and topic treatment) and/or drug suspension.[4548] Since 2008, a possible role of targeted therapies (i.e. antiangiogenic drugs), together with antiresorptives, in developing ONJ has been suggested and investigated.[19,49,50] The so-called anticancer 'targeted therapies' include very different classes of drugs, acting on several cellular signaling pathways, often with 'multi-target' mechanisms. The list of the drugs implicated in the development of ONJ is now increasing, and it will probably continue to do so in the future as several mechanisms are likely to be involved in $\mathrm{ONJ}$, other than disrupting the bone remodeling process. For example, a toxic effect of drugs on soft tissue cells and the local immune system could add to the imbalance between bone resorption and bone formation, which seems the main factor in unraveling the development of BRONJ.

The ONJ-inducing capacity of innovative drugs, such as antiangiogenic and targeted therapies, can be inferred by the following two observations:

(1) The description of ONJ cases in patients treated with such drugs, having not received BPs (or denosumab). These cases serve as the main proof of the existence of ONJ as side effects.

(2) An increase in cases of ONJ in populations of cancer patients undergoing treatment, which included BPs (or denosumab) and the studied drugs.

At this moment, data exist mainly about three agent classes capable of inducing MRONJ:

(i) drugs with main antiangiogenic mechanism (bevacizumab and aflibercept);

(ii) small molecule TKIs (sunitinib, sorafenib, and cabozantinib); (iii) inhibitors of mammalian target of rapamycin (mTOR inhibitors).

The main mechanism of ONJ induced by these novel drugs appears in the antiangiogenic effect, already claimed for BPs (i. e. zoledronic acid), but other mechanisms cannot be excluded.

\subsection{Association of antingiogenic drugs and antiresorptive agents (BPs and denosumab): general considerations}

The first data regarding ONJ cases in patients receiving bevacizumab, the first-developed, and most administered antiangiogenic agent appeared in 2008. Bevacizumab has been approved for several antitumor treatments (including the therapy of colorectal cancer, renal cancer, lung cancer, breast cancer, ovarian cancer, and glioblastoma) and for macular degeneration. The first two reports regarding the association of ONJ with bevacizumab both derived from the Memorial Sloan Kettering Cancer Center (MSKCC) in New York. Estilo et al. [11] published a report in the Journal of Clinical Oncology regarding two cases of osteonecrosis of the jawbone, in one patient with breast cancer and in one patient with glioblastoma, both of whom had been treated with bevacizumab with no history of BP therapy. At the 2008 annual ASCO meeting, McArthur et al. [51] presented an abstract with cases of ONJ in patients treated at MSKCC only with BPs (72 out of 6561, $1.1 \%$ ) or BPs and bevacizumab (8 out of $409,2.0 \%$ ). At the subsequent conference presentation, the data were different, also including ONJ cases that had been observed after treatment only with bevacizumab (probably those reported in the article by Estilo et al.): 76 out of 6534 patients with ONJ had been treated only with BPs, 9 out of 409 had been treated with bevacizumab and BPs, and 2 out of 1711 had only been treated with bevacizumab without BPs.

Suggestive data for an increased ONJ risk have also come from experimental trials in prostate cancer patients by Aragon-Ching et al. [52-54] with very high incidence of ONJ (up to $17 \%$ ) following treatment with zoledronic acid, bevacizumab, and thalidomide.

In subsequent years, reports of ONJ cases after treatment with sunitinib have appeared in the medical literature.[55-62] In a limited cohort, Christodolou et al. observed 5 cases of ONJ among 116 patients who had been receiving BPs from 2007 to 2008, of which 4 had received bevacizumab or sunitinib,[10] thus suggesting an increased risk of ONJ, which is derived from an association of BPs and innovative agents.

The European Medical Agency (EMA) released alerts in 2010 regarding the risks of ONJ after treatment including bevacizumab or sunitinib.[63] Referring to the letters from doctors outlining these alerts, the manufacturers of the drugs declared the following frequency of reported ONJ cases worldwide: 55 out of 800,000 patients treated with bevacizumab, and 27 cases of ONJ out of 101,400 patients receiving sunitinib, most of them also received BPs.

Three large pivotal trials have compared denosumab and zoledronic acid in patients with bone metastases of advanced solid tumors and the results were published separately and on an integrated form with one paper specifically dedicated to ONJ.[45,64] As previously noted,[65] there were 14 cases of 
ONJ in the three trials out of 464 patients (3.0\%) who had been treated with an antiresorptive agent (zoledronic acid or denosumab) and antiangiogenic agents versus 75 cases of ONJ cases out of 5259 patients (1.4\%) receiving zoledronic acid or denosumab and no antiangiogenic agents. This, therefore, suggests a possible higher risk of ONJ from a combination of antiresorptive and antiangiogenic agents. Up to $35 \%$ of advanced renal cell cancer (RCC) patients develop bone metastases, and they are often treated with BPs, mainly zoledronic acid. BRONJ was rarely reported in RCC patients in the first few years of observing ONJ (2003-2006); this was probably due to a short life extension and expectancy and, indeed, there was no mention of RCC patients in the first systematic review reporting ONJ cases published between 2003 and 2005.[66] Few ONJ cases in RCC patients were described after that period.[67] Recently, treatment and prognosis of most metastatic RCC patients have improved, thanks to availability of seven innovative agents: an anti-VEGF monoclonal antibody (bevacizumab), four TKIs, i.e. sorafenib, sunitinib, pazopanib, and axitinib, and two mTOR inhibitors, i.e. temsirolimus and everolimus. Consequently, bone metastatic RCC patients routinely receive targeted therapy as first and further treatment lines, with life expectancy of metastatic RCC patients having almost tripled, leading to a longer exposure to BPs, and thus an increased risk of ONJ. Recent reports have suggested a relatively high ONJ risk in RCC patients after a combination of BPs (mostly zoledronic acid) and antiangiogenic agents (mostly sunitinib).[13,67-69] Bozas and co-workers [68] identified 5 ONJ cases out of 21 RCC patients, who were treated with a combination of BPs and antiangiogenic drugs (23.8\%), with a cumulative hazard ratio for ONJ of $5 \%$ (95\% confidence interval $[\mathrm{Cl}], 0-10 \%)$ and $36 \%(95 \% \mathrm{Cl}, 23-$ 49\%) after 12 and 24 months of treatment with zoledronate, respectively. The authors' conclusion suggests a potential synergy of sunitinib and zoledronic acid in inducing ONJ. In another retrospective study, Beuselinck et al. [69] found that the incidence of ONJ among RCC patients increased up to $10 \%$ when BP and TKIs were administered in combination, with a projected incidence of $17 \%$ of ONJ over 24 months. The authors also hypothesized a possible underestimation of the incidence of ONJ due to the limited survival of patients in the cohort and the short duration of treatment with BP for most patients. A Danish study [13] registered a $29 \%$ incidence of ONJ in RCC patients treated with zoledronic acid and targeted therapy (mainly sunitinib), but the rate decreased to $11 \%$ when oral screening before commencing treatment was recommended. Italian authors collected 44 cases of ONJ with different combinations of BP and targeted agents.[67]

Further literature data seem to strengthen the role of the association of antiresorptives and antiangiogenics in determining ONJ in RCC patients.

First, in the three aforementioned denosumab versus zoledronic acid trials [45,64] of the 89 total adjudicated ONJ patients (treated either with zoledronic acid or denosumab), there were 6 RCC patients $(6.7 \%)$ out of a total number of enrolled RCC patients of 155 . This $6 / 155$ (3.9\%) ONJ frequency in RCC patients is more than twice as high if compared with the entire patient population (1.6\%).[65] Unfortunately, whether those six RCC patients developing ONJ had received antiangiogenic agents or which bone antiresorptive agent had been administered was not discussed in the quoted reports.

Second and in contrast with the initial ONJ reports, the ONJ case series published more recently, included RCC patients in a significant measure, with a frequency ranging from $1 \%$ to $4.5 \%$ of all observed ONJ cases.[67,70]

\subsection{VEGF-mediated antiangiogenic drugs in solid tumors}

Drugs acting against the angiogenesis involving VEGF include bevacizumab and aflibercept.

\subsubsection{Bevacizumab}

Bevacizumab is a monoclonal antibody linking to the angiogenesis growth factor VEGF-A; it was approved for several antitumor treatments (including treatment of colorectal, renal, lung, breast, ovarian, and cervix cancer; glioblastoma), and for macular degeneration treatment. ONJ cases were published in patients receiving bevacizumab since 2008, both on bevacizumab alone and together with BPs (see earlier). ONJ cases after bevacizumab alone were observed in colorectal, breast, lung, parotid gland, and renal cancer patients, and revealed differentiated clinical history and outcome.[12,18,71-76] Furthermore, one case was observed after intravitreous bevacizumab.[77] Some other published cases are notable: one pancreatic cancer patient showed ONJ during treatment with bevacizumab and sorafenib [78] and one renal cell cancer patient had received bevacizumab plus temsirolimus [79]; another renal cell cancer patient developed ONJ during bevacizumab treatment, after previous treatments with sunitinib and temsirolimus.[80] To note, one glioblastoma patient developed a severe complication after a single bevacizumab infusion [81]compatible with complicated ONJ. Besides case reports, few data are available about frequency of bevacizumabrelated ONJ. We have already reported the initial experience from Memorial Sloan Kettering Cancer Center.[51] In 2010, a retrospective study on breast cancer patients revealed a very low risk after bevacizumab alone $(0.3-04 \%)$ in comparison with the combination of bevacizumab and BPs (0.9-2.4\% range) within a large trial population [12] However, only ONJ cases with frank bone exposure were registered in that analysis, and median follow-up of groups ranged only between 10 and 19 months. Were ONJ cases in bevacizumab-treated patients somewhat different from BRONJ cases? In a single center experience report, Ngamphaiboon et al. [82] compared 7 ONJ cases observed after BPs and bevacizumab treatment and 20 cases after BPs without bevacizumab, all observed at the Roswell Park Cancer Institute, without relevant differences. Lescaille et al. [83]on the contrary found in their 42 ONJ case population that bevacizumab was associated to earlier onset, higher risk of spontaneous developing (without trigger) and higher number of jaw lesions.

\subsubsection{Aflibercept}

Aflibercept is another agent potentially capable of determining ONJ. Aflibercept, the so-called 'VEGF trap', is an antiangiogenic agent with mechanism of action different from that of bevacizumab, being a soluble decoy VEGF receptor. 
Aflibercept has been approved for treatment of advanced colorectal cancer and has been largely evaluated as treatment of other cancer types. ONJ cases in patients receiving aflibercept are present in an integrated safety database reported by North American and European agencies in 2012[84]: out of six ONJ cases among aflibercept-treated patients three patients also received BPs. Two ONJ cases were observed within the pivotal VELOUR (VEGF-trap with irinotecan in colorectal cancer after failure of oxaliplatin) trial,[85] of which only one case of treatment with BPs was found.[84] Another ONJ case was observed in an exploratory trial of aflibercept on melanoma patients, but the patient was also irradiated to head and neck area.[86] One more case has been recently reported.[87] As aflibercept has been and will be probably more largely administered to colon cancer patients, a large population of patients partially pretreated with bevacizumab and more cases are reasonably to be expected.

\subsection{TKIs}

TKIs are drugs with differentiated effects (including antiangiogenic ones) [88]: they block tumor growth and inhibit angiogenesis through multi-target mechanisms. A large number of TKIs have been registered as active drugs and authorized for clinical practice, or are under evaluation in phase II and phase III trials. At our best knowledge, no cases of ONJ have been published among patients participating to trials that conducted to registration of approved agents, such as sunitinib, sorafenib, pazopanib, axitinib, gefitinib, erlotinib, crizotinib, ceritinib, imatinib, dabrafenib, dasatinib, regorafenib, etc., in a series of cancer types (renal cell cancer, soft-tissue sarcoma, lung cancer, pancreatic neoplasms, gastrointestinal stromal tumors (GISTs), hepatocellular cancer, melanoma, thyroid cancer, colon cancer, etc.). Nevertheless, we have to remind that no cases of ONJ had been initially reported even in pivotal papers that conducted large introduction in clinics of BPs determining BRONJ (alendronate, risedronate, ibandronate, pamidronate, zoledronic acid) in 1990s and in the beginning of this century; and neither ONJ cases were present in reports of first trials with bevacizumab and sunitinib. Consequently, we should wait for observation in large confirmatory studies, and above all in clinical practice population out of clinical trials (i.e. observational studies and drug surveillance system reports), with adequately long follow-up time, before excluding capacity of TKIs other than sunitinib to determine ONJ without antiresorpive agents.

\subsubsection{Sunitinib}

Most of the data available for TKI-related ONJ cases are linked to sunitinib, a drug-inducing stomatitis and dental side effect in a large proportion of patients.[89] Besides initial reports of patients also treated with BPs, $[10,55,56,58,62,90]$ sunitinib appeared as the only agent administered in some RCC patients suffering of ONJ [59-61] Furthermore, sunitinib was associated to BPs in numerous ONJ cases in RCC population, [67] with an incidence higher than expected when a denominator was reported. [13,57,69] However, Smidt-Hansen reported the possibility of decreasing the ONJ risk after adopting preventive measures (as well as recommended in general BP-treated population).

\subsubsection{Other TKIs}

Data about sorafenib-related ONJ risk are scarce, and are absent for those about possible role of pazopanib and axitinib. Sorafenib treatment was present, as only targeted therapy or in sequence with others, in the treatment history of RCC patients also receiving BPs.[67,69] One case report has been published about MRONJ observed in a patient with medullary thyroid cancer treated with cabozantinib without BP treatment history [91]; three ONJ cases had been reported in the original pivotal trial by Elisei et al., [92] but data about eventual BP treatment are missing.

\section{4. mTOR inhibitors}

Inhibitors of mTOR pathway are largely administered as immunosuppressive agents as well as anticancer drugs.[93] Inhibitors of mTOR (everolimus and temsirolimus) are agents approved for anticancer treatment (renal cell cancer, breast cancer, and pancreatic neuroendocrine tumors) and prevention of solid organ rejection. As mTOR signaling is linked to the VEGF pathway, ONJ cases could be expected after treatment with those drugs.

\subsubsection{Temsirolimus}

Temsirolimus is approved for RCC; one case of ONJ in an RCC patient never treated with BPs has been observed after therapy with temsirolimus alone.[67,94] Other two cases were observed during treatment with sunitinib and temsirolimus [62] and bevacizumab and temsirolimus.[79] One patient developed ONJ during treatment with ridaforolimus, an experimental agent, and pamidronate.[67]

\subsubsection{Everolimus}

Everolimus is an mTOR inhibitor experimented in several cancer types and some rare diseases.[95] Everolimus is administered for several years for advanced renal cell cancer treatment: sparse cases of ONJ were reported in literature after treatment with everolimus together with BPs in RCC patients.[13,14,67,96] Another case has been described after 3-year treatment with everolimus in a medullary thyroid cancer previously pretreated many years before with zoledronic acid.[15] Everolimus has been more recently studied in advanced breast cancer to revert endocrine resistance and obtained registration in association with exemestane, an endocrine agent. In the pivotal BOLERO-2 trial,[97,98] ONJ has been observed in 2 out of 482 patients in the experimental (everolimus-exemestane) arm and 1 out 238 in the control (exemestane alone) arm, with one of the three pretreated with BPs, as reported by Gnant et al. in a corollary paper [99]; however, no ONJ case was linked to the trial treatment by the local investigator. Finally, no ONJ cases have been reported in other trials with everolimus, but there was a notable heterogeneity in reporting side effects involving oral cavity, where 'stomatitis' (the most common side effect of everolimus) also included gingival swelling and jaw pain. [100] Furthermore, in one everolimus trial, a 3\% incidence of 
unexpected 'jaw pain' was classified among 'musculoskeletal and connective tissue disorders'.[100] As reported by Aapro et al. [101] about breast cancer patients receiving everolimus, 'in theory, ONJ is part of the differential diagnosis at the presentation of a stomatitis event. Although the denuded bone area is easily distinguished from superficial mucosal lesions on the gum, preliminary symptoms, such as pain are observed in both clinical conditions'. Therefore, in consideration of possible cases of ONJ without frank bone exposure, further careful observation data about oral side effect in patients receiving everolimus (with/without BPs or denosumab) are recommended to avoid ONJ late or missed diagnosis.

\subsection{Antiangiogenic drugs in myeloma}

Thalidomide and analogs (lenalidomide and pomalidomide) have been largely adopted in the treatment of myeloma patients, a population largely receiving BPs and consequently at risk of BRONJ. Thalidomide and analogs have antiangiogenesis effect with not completely known mechanism, and consequently they might increase ONJ risk in myeloma patients; however, in spite of largely treated populations on trials, in practice there is no sufficient data to confirm or deny this hypothesis.[40]

\subsection{Other drugs}

Very recently, a case of ONJ was observed in a melanoma patient receiving immunotherapy with ipilimumab at the Memorial Sloan Kettering Cancer Center in New York [102]; no history of BP, denosumab, or antiangiogenic treatment was present. The ONJ case shortly resolved after antibiotic therapy and removal of bone sequestrum.

\subsection{Specific characteristics of ONJ after antiangiogenic and other non-antiresorptive drugs}

Further data are needed about the possibility that ONJ related to antiangiogenic agents show predominantly a clinical history different from that - already heterogeneous - of BRONJ: some papers reported about consecutive episodes of painful jaw infections,[56] with self-limiting history after antibiotic treatment and drug suspension [73]and without bone exposure or with limited exposure in time and in largeness. Imaging studies (particularly by computed tomography (CT) or bone scan scintigraphy) in case of symptomatic oral health discomfort in cancer patients receiving antiangiogenic and targeted therapies should be of great importance, as a large part of ONJ cases might escape the classical definition (requiring 8-week lasting bone exposure). Imaging studies of ONJ cases related to targeted therapies in comparison to those of BRONJ and denosumab-related ONJ cases are lacking at this moment.

\section{Conclusion}

As every year novel drugs with antiangiogenic properties and multi-target mechanisms are introduced in the anticancer therapeutic armamentarium, clinicians and patients have to give high attention to oral health problems during treatment with new agents, in order to distinguish between stomatitis, a very common side effect of most of these drugs, and potential signs of early ONJ, with or without bone exposure. As $20-30 \%$ of ONJ cases escape the classical definition (requiring 8-week lasting bone exposure), $[24,35,36]$ great attention should be paid to imaging studies (particularly by $C T$ or bone scan scintigraphy) in order to avoid underestimation of MRONJ.

\section{Expert opinion}

Some key-questions are consequently in front of clinical oncologists and other caregivers.

(1) How many patients suffer or will suffer ONJ after antiangiogenic agents, with or without concomitant administration ofBPs or denosumab?

We do not know exactly the burden of this new phenomenon. Safety surveillance systems will be helpful and should report case numbers likely higher than those extracted by literature reports. Unfortunately, propensity of physicians to register adverse drug reactions is far to be optimal.[103] Obviously, prospective collection and registration of data could be of even higher value, even if not uniformly applicable.[30] Furthermore, any data are to be interpreted with caution due to difficult adjudication of the adverse drug reaction to one drug or to another in cancer patients often receiving a large number of drugs simultaneously or in sequence.

For example, in Italy a large number of ONJ cases (several thousands) have been observed, and Italian physicians and dentists had contributed largely to knowledge and awareness of the disease with conferences, papers, and publications,[104] but only a minority of cases has been notified to the Italian Safety Drug Agency (AIFA). In march 2015, only 1513 cases of ONJ related to bisphosphonates in 10 years had been reported to AIFA; at that time, the AIFA database also contained 27 cases of ONJ related to denosumab, 16 related to bevacizumab, 20 to sunitinib, and 1 to sorafenib (AIFA, personal communication). This phenomenon of under-reporting is observed in other industrialized countries.[70,105] Finally, available data relating to $\mathrm{ONJ}$ in patients treated with innovative anticancer drugs are scarce but on the increase. There is a high risk of underestimation due to a lack of awareness, and restrictive or inadequate diagnostic criteria, together with the need to improve the defining of any distinguished feature in the diagnosis, prevention, and therapy of ONJ, as associated with nonantiresorptive agents.

(2) Who are the patients at higher risk of ONJ during or after treatment including antiangiogenic agents?

From an epidemiological point of view, till now breast and prostate cancer patients with bone metastases and myeloma patients have been the main ONJ populations due to more frequent and more prolonged BP and denosumab treatments. With reference to antiangiogenic treatments, renal cell cancer patients were one of the first at-risk populations due to 
enlarged adoption of targeted therapies, alone or with BPs, [67] but the extension of use of aforementioned drugs in some more patient populations could give higher probability of ONJ onset. It is the case of patients suffering colon cancer (receiving bevacizumab, aflibercept, and other antiangiogenic drugs), lung cancer (bevacizumab, TKIs), and ovarian cancer (in future largely treated with bevacizumab). Furthermore, availability of inclusion of patients in several trials or extended access programs with novel drugs or treatment protocols could enlarge the risk; it is not infrequent that a single patient could receive more biological agents in sequence, on and off-label, especially if achieved survival is longer than usual. It is reasonable that longer drug(s) treatment duration and longer survival are elements associated with higher ONJ rates.

(3) Are clinical features and outcome of ONJ related to antiangiogenic drugs and other 'targeted therapy' similar to or different from that observed after bisphosphonate or denosumab treatment?

With respect to the clinical features, in our opinion there is no definitive answer yet for a series of reasons. First, it is difficult to attribute the adverse drug reaction (ONJ) exclusively to one drug or to another in cancer patients often receiving a large number of drugs simultaneously or in sequence. After observing cases of ONJ where patients were treated with antiangiogenic agents with concomitant or previous antiresorptive drugs, the clinical findings have been quite similar to those in patients taking BPs even after shorter treatment durations and/or lower cumulative dosages, hypothesizing a precipitating role for antiangiogenic agents: for example, Lescaille et al. reported the onset of ONJ after a median of 12.4 months of bevacizumab and zoledronate in comparison with a median 22.9 months in patients who received only zoledronate.[83] Description of ONJ literature cases after antiangiogenic agents without history of antiresorptive drugs is variable; the ONJ clinical history (particularly presence or not of bone exposure, duration of exposure, and shortterm improvement or worsening) could be probably related to drug effects on soft tissues (gingival) and influenced by drug administration schedule. A peculiarity of these new classes of drugs, different from BPs, is their pharmacodynamics (without bone accumulation) and shorter half-life (e.g. around 40-50 days for bevacizumab; 7 days for sunitinib, aflibercept, everolimus); as a consequence, it is possible, in case of ONJ onset with accordance between oncologist and dental care specialist, to plan their suspension in view of a resolution of ONJ event.

(4) How to label this aforementioned ONJ form and is it hard to diagnose it?

Since 2003, ONJ appeared as a BP-related class effect, and the term BRONJ was widespread. Recent studies have shown that denosumab is also capable of inducing ONJ as well as antiangiogenic agents and other 'targeted therapies', consequently, the core ' $\mathrm{ONJ}$ ' is once again largely used alone; alternatively, the term MRONJ has been recently introduced. [31] With respect to the diagnostic work-up, the main problem is the possible under-diagnosing of ONJ cases without bone exposure. A recent multicenter study [24] has demonstrated that the use of the traditional AAOMS [16,37] and ASBMR case definition [17] induces clinicians up to a quarter of undiagnosed BRONJ cases. These cases regard those patients who do not have any bony exposure but they do have unexplained pain in the jaws, fistula, loose teeth, swelling, and occasional pathological mandibular fracture. Thus, in order to avoid any underestimation of ONJ in general, care should be taken with respect to the definition adopted, and to the use of the imaging studies (particularly by $\mathrm{CT}$ or bone scan scintigraphy) of symptomatic oral health discomfort in cancer patients, who are already receiving antiangiogenic and targeted therapies in combination with antiresorptives or alone. In 2014, the AAOMS [31] admitted the existence of a non-exposed ONJ disease, conceding for the first time the definition of ONJ at least for cases without bone exposition and with bone probing via sinus/fistula tracts, but the task force did not change the old statement on staging (including the description of a 'stage 0 ' conflicting with the definition). Notwithstanding this development, many studies in this field still continue, unreasonably (the authors contend), to adopt the 2009 AAOMS definition.[37] In the report by Saad et al. [45] of the cumulative data of the three denosumab versus zoledronate trials, 89/ 5723 ONJ cases (1.5\%) were adjudicated according to strict AAOMS criteria, but $276 / 5723(4.8 \%)$ cases were suspected for ONJ signs or symptoms ('potential ONJ'): it is natural to doubt about possible missed ONJ diagnosis. On the other hand, the AAOMS definition of ONJ has been recently found to be 'unfit for purpose' in the spontaneous reporting database: by authors its use should be avoided, leading to the exclusion of over $70 \%$ of cases from the database.[106] An effective improvement in the ONJ staging was demonstrated by CT.[28,30]

Finally, it appears particularly challenging for the determination of early imaging (CT) signs of bone alteration, already described in BP-related ONJ [24,36]; moreover, this kind of imaging could be potentially valuable not only in order to facilitate recognition of ONJ without bone exposure but also to make easier the differential diagnosis between ONJ and drug-related long-lasting stomatitis, a possible problem after some drug treatment (e.g. everolimus).[101] Scintigraphy bone scan and positron emission tomography could be of value in differential diagnosis, but their cost-effectiveness (if not already made due to cancer disease staging) seems not warranted.

(5) What dental preventive measures could be taken in patients receiving antiangiogenic and targeted therapies?

As of now, there is not a validated user guide for these classes of drugs. But, it is believed that the major local risk factors remain, at this moment, the same dental history features as already known for patients receiving BPs and denosumab: i.e. tooth extraction and alveolar surgery, implants, illfitting dentures, and poor oral health. Hence, in general, the preventive protocols already adopted for the antiresorptive agents [40] might be used for these new drugs. These agents share a characteristic with denosumab, that is the option of the drug holiday in case of surgical dental procedures. The aim of this is to reduce the risk of an ONJ event after a tooth extraction, or endodontic surgery, or prosthodontic surgery. 
Table 1. Drug holiday suggested by the authors in the case of dental surgery based on half-life of the drugs.

\begin{tabular}{lcc}
\hline Molecule & $\begin{array}{c}\text { Start of drug } \\
\text { holiday }\end{array}$ & \multicolumn{1}{c}{ Retaking } \\
\hline Bevacizumab & $\begin{array}{c}40-50 \text { days prior } \\
\text { to surgery }\end{array}$ & $\begin{array}{c}\text { A minimum of } 30-45 \text { days } \\
\text { postsurgery } \\
\text { (clinical and radiological } \\
\text { healing are necessary) }\end{array}$ \\
$\begin{array}{c}\text { Sunitinib, aflibercept, } \\
\text { and everolimus }\end{array}$ & $\begin{array}{c}7 \text { days prior to } \\
\text { surgery }\end{array}$ & $\begin{array}{c}\text { A minimum of } 30-45 \text { days } \\
\text { postsurgery } \\
\text { (the clinical and radiological } \\
\text { healing are necessary) }\end{array}$ \\
\hline
\end{tabular}

However, the authors wish to specify that there is no robust literature relating to this topic; on the basis of the half-life of the molecules involved, it could be useful to adopt a drug holiday scheme for cancer patients. The authors suggest the drug holiday time as indicated in Table 1.

In summary, the authors believe that ONJ is a clinically important, potentially painful, and debilitating condition, which can significantly affect the QoL of cancer patients. New patient populations are at risk of ONJ. The list of nonantiresorptive drugs, which are implicated in the development of ONJ, is growing and will probably continue to do so as several mechanisms are likely to be involved in ONJ, other than those disrupting the bone remodeling process. Moreover, the clinical aspects and especially the prognosis of ONJ associated with these new drugs seem to be unlike those used in BRONJ. Accordingly, recommendations for diagnosis, prevention, and treatment, which have already been developed and currently in use for BRONJ, should be adopted, but critically and cautiously, basing treatment on an individual risk assessment. Due to all these, a higher awareness of physicians (including oncologists and pathologists), dentists, nurses, and patients is the main way to obtain more information about drug-related ONJ, so that adequate measures could be adopted to minimize this potentially severe adverse drug reaction.

\section{Declaration of interests}

The authors have no relevant affiliations or financial involvement with any organization or entity with a financial interest in or financial conflict with the subject matter or materials discussed in the manuscript. This includes employment, consultancies, honoraria, stock ownership or options, expert testimony, grants or patents received or pending, or royalties.

\section{References}

Papers of special note have been highlighted as:

(•) of interest

(•.) of considerable interest

1. Hoff A, Toth BB, Altundag K, et al. The frequency and risk factors associated with osteonecrosis of the jaw in cancer patients treated with intravenous bisphosphonates. J Bone Miner Res. 2008;23:826836.

2. Adamo V, Caristi N, Saccà MM, et al. Current knowledge and future directions on bisphosphonate-related osteonecrosis of the jaw in cancer patients. Expert Opin Pharmacother. 2008;9(8):1351-1361.

3. Durie BGM, Katz M, Crowley J. Osteonecrosis of the jaws and bisphosphonates. N Engl J Med. 2005.
.. This reference is of particular importance as it is one of the first works that conducts a detailed overview on the risk of ONJ in various diseases in conjunction with the use of bisphosphonates.

4. Miksad RA, Lai KC, Dodson TB, et al. Quality of life implications of bisphosphonate-associated osteonecrosis of the jaw. Oncologist. 2011;16(1):121-132.

-. This study represents one of the first evidence in which it is explained how the ONJ may impact the quality of life of cancer patients.

5. Stewart ST, Lenert L, Bhatnagar V, et al. Utilities for prostate cancer health states in men aged 60 and older. Med Care. 2005;43(4):347355.

6. Loeb S, Roehl KA, Helfand BT, et al. Complications of open radical retropubic prostatectomy in potential candidates for active monitoring. Urology. 2008;72(4):887-891.

7. Setabutr D, Hales NW, Krempl GA. Necrotizing fasciitis secondary to bisphosphonate-induced osteonecrosis of the jaw. Am J Otolaryngol [Internet]. 2010 [cited 2009 Mar 26];31(2):127-129. doi:10.1016/j.amjoto.2008.11.002.

8. Randi L, De Martino I, Fasciolo A, et al. Osteonecrosis of jaw (ONJ): sometimes a life-threatening event. Literature review and two cases. Ann Stomatol (Roma). 2014;5(2 Suppl): 33-34. Available from: http://www.ncbi.nlm.nih.gov/pmc/articles/PMC4377653/

9. Mondello $\mathrm{P}$, Pitini V, Arrigo $C$, et al. Necrotizing fasciitis as a rare complication of osteonecrosis of the jaw in a patient with multiple myeloma treated with lenalidomide: case report and review of the literature. Springerplus. 2014;3: 123. doi:10.1186/2193-1801-3-123. eCollection 2014

10. Kaehling C, Streckbein P, Schmermund D, et al. Lethal cervical abscess following bisphosphonate related osteonecrosis of the jaw. J Craniomaxillofac Surg. 2014;42(7):1203-1206. doi:10.1016/j. jcms.2014.02.009.

11. Estilo $C L$, Fornier $M$, Farooki $A$, et al. Osteonecrosis of the jaw related to bevacizumab. J Clin Oncol. 2008;26:4037-4038.

-. It is the first case report on oil combined with the use of antiVEGF agents.

12. Guarneri V, Miles D, Robert N, et al. Bevacizumab and osteonecrosis of the incidence and association with bisphosphonate therapy in three large prospective trials in advanced breast cancer. Breast Cancer Res Treat. 2010;122(1):181-188.

13. Smidt-Hansen T, Folkmar TB, Fode K, et al. Combination of zoledronic acid and targeted therapy is active but may induce osteonecrosis of the jaw in patients with metastatic renal cell carcinoma. J Oral Maxillofac Surg. 2013;71(9):1532-1540.

14. Giancola F, Campisi G, Russo LL, et al. Osteonecrosis of the jaw related to everolimus and bisphosphonate: a unique case report?. Ann Stomatol. 2013;4(Suppl 2):20-21.

.- This case report is the first evidence of ONE associated with the use of inhibitors of mammalian target of rapamycin (mTOR).

15. Kim DW, Jung YS, Park HS, et al. Osteonecrosis of the jaw related to everolimus: a case report. Br J Oral MaxillofacSurg. 2013;4(Suppl 2):20-21.

16. Advisory Task Force on Bisphosphonate-Related Ostenonecrosis of the Jaws, American Association of Oral and Maxillofacial Surgeons. American Association of Oral and Maxillofacial Surgeons position paper on bisphosphonate-related osteonecrosis of the jaws. J Oral Maxillofac Surg. 2007;65(3):369-376.

17. Khosla, S., Burr D, Cauley J et al. Bisphosphonate-associated osteonecrosis of the jaw: report of a task force of the American Society for Bone and Mineral research. J Bone Miner Res. 2007;22(10):1479-1491.

18. Estilo $C L$, Fornier $M$, Farooki $A$, et al. Osteonecrosis of the jaw related to bevacizumab. J Clin Oncol. 2008;26:4037-4038.

19. Troeltzsch M, Woodlock T, Kriegelstein S, et al. Physiology and pharmacology of nonbisphosphonate drugs implicated in osteonecrosis of the jaw. J Can Dent Assoc. 2012;78:c85.

20. Junquera L, Gallego L. Nonexposed bisphosphonate-related osteonecrosis of the jaws: another clinical variant? J Oral Maxillofac Surg. 2008;66(7):1516-1517. 
21. Mawardi $\mathrm{H}$, Treister N, Richardson $\mathrm{P}$, et al. Sinus tracts-an early sign of bisphosphonate-associated osteonecrosis of the jaws? J Oral Maxillofac Surg. 2009;67(3):593-601.

22. Ruggiero SL, Dodson TB, Assael LA, et al. American Association of Oral and Maxillofacial Surgeons position paper on bisphosphonaterelated osteonecrosis of the jaws-2009 update. J Oral Maxillofac Surg. 2009;67(suppl 1):2-12.

23. Colella G, Campisi G, Fusco V. American Association of Oral and Maxillofacial Surgeons position paper: bisphosphonate-related osteonecrosis of the jaws-2009 update: the need to refine the BRONJ definition. J Oral Maxillofac Surg. 2009;67(12):2698-2699.

24. Fedele S., Porter SR, D'Aiuto F, et al. Nonexposed variant of bisphosphonate-associated osteonecrosis of the jaw: a case series. Am J Med. 2010;123(11):1060-1064.

25. Yarom N, Fedele S, Lazarovici TS, et al. Is exposure of the jawbone mandatory for establishing the diagnosis of bisphosphonaterelated osteonecrosis of the jaw? J Oral Maxillofac Surg. 2010;68 (3):705.

26. Hutchinson M, O'Ryan F, Chavez V, et al. Radiographic findings in bisphosphonate-treated patients with stage 0 disease in the absence of bone exposure. J Oral Maxillofac Surg. 2010;68 (9):2232-2240.

27. Patel S, Choyee S, Uyanne J, et al. Non-exposed bisphosphonate related osteonecrosis of the jaw: a critical assessment of current definition, staging, and treatment guidelines. Oral Dis. 2012;18 (7):625-632.

28. Bedogni A, Fusco V, Agrillo A, et al. Learning from experience. Proposal of a refined definition and staging system for bisphosphonate- related osteonecrosis of the jaw (BRONJ). Oral Dis. 2012;18(6):621-623.

29. Campisi G, Lo Russo L, Agrillo A, et al. BRONJ expert panel recommendation of the Italian Societies for Maxillofacial Surgery (SICMF) and Oral Pathology and Medicine (SIPMO) on bisphosphonate-related osteonecrosis of the jaws: risk assessment, preventive strategies and dental management. Italian J Maxillofac Surg. 2011;22(2):103-124.

. This paper emphasizes resolutely conditions non-exposed ONJ.

30. Schiodt M, Reibel J, Oturai P, et al. Comparison of nonexposed and exposed bisphosphonate-induced osteonecrosis of the jaws: a retrospective analysis from the Copenhagen cohort and a proposal for an updated classification system. Oral Surg Oral Med Oral Pathol Oral Radiol. 2014;117(2):204-213.

31. Ruggiero SL, Dodson TB, Fantasia J, et al. American Association of Oral and Maxillofacial Surgeons. American Association of Oral and Maxillofacial Surgeons position paper on medication-related osteonecrosis of the jaw-2014 update. J Oral Maxillofac Surg. 2014;72 (10):1938-1956. doi:10.1016/j.joms.2014.04.031.

32. Khan AA, Morrison A, Hanley DA, et al. Diagnosis and management of osteonecrosis of the jaw: a systematic review and international consensus. J Bone Miner Res. 2015;30(1):3-23.

33. Otto $S$, Marx RE, Tröltzsch M, et al. Comments on "diagnosis and management of osteonecrosis of the jaw: a systematic review and international consensus". j bone Miner Res. 2015;30:1113-1115. doi:10.1002/jbmr.2525.

34. Fusco V, Galassi C, Berruti A, et al. Osteonecrosis of the jaw after zoledronic acid and denosumab treatment. J Clin Oncol. 2011;29 (17):e521-e522;author reply e523-4. doi:10.1200/JCO.2011.35.1551.

.. In this study, the definition of ONJ during the use of agents that inhibit osteoclast activity is underlined with accuracy.

35. Fedele S, Bedogni G, Scoletta $M$, et al. Up to a quarter of patients with osteonecrosis of the jaw associated with antiresorptive agents remain undiagnosed. $\mathrm{Br} J$ Oral Maxillofac Surg. 2015;53(1):13-17.

36. Bedogni A, Fedele S, Bedogni G, et al. Staging of jaw osteonecrosis requires computed tomography for accurate definition of the extent of bony disease. Br JMaxillofac Surg. 2014;52:603-608.

37. Ruggiero SL. Bisphosphonate-related osteonecrosis of the jaw (BRONJ): initial discovery and subsequent development. J Oral Maxillofac Surg. 2009;67(5 Suppl):13-18. doi:10.1016/j.joms.2008.10.005.
38. Allen MR, Burr DB. The pathogenesis of bisphosphonate-related osteonecrosis of the jaw: so many hypotheses, so few data. J Oral Maxillofac Surg. 2009;67(suppl 5):61-70.

39. Bertoldo F, Santini D, Lo Cascio V. Bisphosphonates and osteomyelitis of the jaw: a pathogenic puzzle. Nat Clin Pract Oncol. 2007;4:711-721.

- Although etiology of BRONJ has not yet been completely elucidated, this work sheds light on origins multifactorial agents that conduct to the ONJ.

40. Campisi G, Fedele F, Fusco V, et al. Epidemiology, clinical manifestations, risk reduction and treatment strategies of jaw osteonecrosis in cancer patients exposed to antiresorptive agents. Future Oncol. 2014;10:257-275.

- This paper explains how the amount of biological and animal model data is growing but there is currently no single phenomenon explaining the variety of $\mathrm{ONJ}$, which has arisen after intravenous or oral BP treatment.

41. Santini D, Galluzzo S, Vincenzi B, et al. New developments of aminobisphosphonates: the double face of Janus. Ann Oncol. 2007;18(suppl6):vi164-vi167.

.. This work supports the hypothesis on the antiangiogenic activity of BPs as one of the main determinants of the onset of ONJ.

42. Vincenzi B, Napolitano A, Zoccoli A, et al. Serum VEGF levels as predictive marker of bisphosphonate-related osteonecrosis of the jaw. J Hematol Oncol. 2012;5:56.

43. Arduino PG, Menegatti $E$, Scoletta $M$, et al. Vascular endothelial growth factor genetic polymorphisms and haplotypes in female patients with bisphosphonate-related osteonecrosis of the jaws. J Oral Pathol Med. 2011;40(6):510-515. doi:10.1111/ j.16000714.2010.01004.x.

44. Allegra A, Alonci A, Penna G, et al. Bisphosphonates induce apoptosis of circulating endothelial cells in multiple myeloma patients and in subjects with bisphosphonate-induced osteonecrosis of the jaws. Acta Haematol. 2010;124(2):79-85. doi:10.1159/000313787.

45. Saad F, Brown JE, Poznak CV, et al. Incidence, risk factors, and outcomes of osteonecrosis of the jaw: integrated analysis from three blinded active-controlled phase III trials in cancer patients with bone metastases. Ann Oncol. 2012;23:1341-1347.

46. Toole J. Osteonecrosis of the jaws. Rheumatology (Oxford). 2015;54 (10):1755-1756. doi:10.1093/rheumatology/kev094. Epub 2015 Apr 14.

47. Aghaloo TL, Dry SM, Mallya S, et al. Stage 0 osteonecrosis of the jaw in a patient on denosumab. J Oral Maxillofac Surg [Internet]. 2014 [cited 2014 Jan 4];72(4):702-716. doi:10.1016/j. joms.2013.09.008.

48. Niibe K, Ouchi T, Iwasaki R, et al. Osteonecrosis of the jaw in patients with dental prostheses being treated with bisphosphonates or denosumab. J Prosthodont Res. 2015;59(1):3-5. doi:10.1016/j.jpor.2014.08.001.

49. Yarom N, Elad S, Madrid C, et al. Osteonecrosis of the jaws induced by drugs other than bisphosphonates-a call to update terminology in light of new data. Oral Oncol. 2010;46:e1.

50. Hamadeh IS, Ngwa BA, Gong Y. Drug induced osteonecrosis of the jaw. Cancer Treat Rev [Internet]. 2015 [cited 2015 Apr 21];41 (5):455-464. doi:10.1016/j.ctrv.2015.04.007.

51. McArthur HL, Estilo C, Huryn J, et al. Osteonecrosis of the jaw (ONJ) among intravenous (IV) bisphosphonate- and/or bevacizumab-treated patients (pts) at Memorial Sloan-Kettering Cancer Center (MSKCC). J Clin Oncol. 2008 ASCO Annual Meeting Proceedings (Post-Meeting Edition). Vol 26, No 15S (May 20 Supplement). 2008;9588. Available from: http://meeting.ascopubs.org/cgi/con tent/abstract/26/15_suppl/9588

52. Aragon-Ching JB, Ning YM, Latham L, et al. Osteonecrosis of the jaw (ONJ) in androgen-independent prostate cancer (AIPC) patients receiving ATTP (bevacizumab, docetaxel, thalidomide, and prednisone). J Clin Oncol. 2007;25:19594.

53. Aragon-Ching JB, Dahut WL. Osteonecrosis of the jaw and the use of antiangiogenic agents: just an association? Oncologist. 2008;13:1314. 
54. Aragon-Ching JB, Ning YM, Chen C, et al. Higher incidence of Osteonecrosis of the jaw (ONJ) in patients with metastatic castration resistant prostate cancer treated with anti-angiogenic agents. Cancer Invest. 2009;27:221-226.

55. Ayllon J, Launay-Vacher V, Medioni J, et al. Osteonecrosis of the jaw under bisphosphonate and antiangiogenic therapies: cumulative toxicity profile? Ann Oncol. 2009;20:600-601.

56. Brunello A, Saia G, Bedogni A, et al. Worsening of osteonecrosis of the jaw during treatment with sunitinib in a patient with metastatic renal cell carcinoma. Bone. 2009;44:173-175.

57. Bozas G, Roy A, Ramasamy V, et al. Osteonecrosis of the jaw after a single bisphosphonate infusion in a patient with metastatic renal cancer treated with sunitinib. Onkologie. 2010;33:321-323.

58. Hoefert S, Eufinger H. Sunitinib may raise the risk of bisphosphonate-related osteonecrosis of the jaw: presentation of three cases. Oral Surg Oral Med Oral Pathol Oral Radiol Endod. 2010;110:463469.

59. Koch FP, Walter $C$, Hansen $T$, et al. Osteonecrosis of the jaw related to sunitinib. Oral Maxillofac Surg. 2011;15:63-66.

60. Nicolatou-Galitis O, Migkou M, Psyrri A, et al. Gingival bleeding and jaw bone necrosis in patients with metastatic renal cell carcinoma receiving sunitinib: report of 2 cases with clinical implications. Oral Surg Oral Med Oral Pathol Oral Radiol. 2012;113:234-238.

61. Fleissig Y, Regev E, Lehman H. Sunitinib-related osteonecrosis of jaw: a case report. Oral Surg Oral Med Oral Pathol Oral Radiol. 2012;113:e1-e3.

62. Agrillo A, Nastro Siniscalchi E, Facchini A, et al. Osteonecrosis of the jaws in patients assumine bisphosphonates and sunitinib: two case reports. Eur Rev Med Pharmacol Sci. 2012;16:952-957.

63. MHRA. Bevacizumab and sunitinib: risk of osteonecrosis of the jaw. Drug Safety Update. 2011. Available from: http://www.mhra.gov. uk/Safetyinformation/DrugSafetyUpdate/CON105745

64. Lipton A, Fizazi K, Stopeck AT, et al. Superiority of denosumab to zoledronic acid for prevention of skeletal-related events: a combined analysis of 3 pivotal, randomised, phase 3 trials. Eur J Cancer. 2012;48:3082-3092.

65. Fusco V, Bedogni A, Campisi G. Osteonecrosis of the jaw (ONJ) in renal cell cancer patients after treatment including zoledronic acid or denosumab. Support Care Cancer. 2014;22:1737-1738.

66. Woo SB, Hellstein JW, Kalmar JR. Systematic review: bisphosphonates and osteonecrosis of the jaws. Ann Intern Med. 2006;144:753-761.

67. Fusco V, Porta C, Saia G, et al. Osteonecrosis of the jaw in patients with metastatic renal cell cancer treated with bisphosphonates and targeted agents: results of an Italian multicenter study and review of the literature. Clin Genitourin Cancer. 2015;13(4):287-294. doi:10.1016/j.clgc.2014.12.002.

-. This work, in contrast to the earlier report, highlights the incidence of ONJ in patients with renal cell cancer, giving substantive hypothesis of how many cases have been underestimated in the past.

68. Bozas G, Allgar V, Greenwood G, et al. Osteonecrosis of the jaw in patients treated with sunitinib and zoledronic acid (abstract e15116). J Clin Oncol. 2011. Available from: http://meetinglibrary. asco.org/content/82466-102

69. Beuselinck B, Wolter P, Karadimou A, et al. Concomitant oral tyrosine kinase inhibitors and bisphosphonates in advanced renal cell carcinoma with bone metastases. Br J Cancer. 2012;107:16651671.

70. Gabbert Tl, Hoffmeister B, Felsenberg D. Risk factors influencing the duration of treatment with bisphosphonates until occurrence of an osteonecrosis of the jaw in 963 cancer patients. J Cancer Res Clin Oncol. 2014; 141; 749-58

71. Greuter S, Schmid F, Ruhstaller T, et al. Bevacizumab-associated osteonecrosis of the jaw. Ann Oncol. 2008;19:2091-2092.

72. Guarneri V, Miles D, Robert N, et al. Bevacizumab and osteonecrosis of the jaw: incidence and association with bisphosphonates therapy in three large prospective trials in advanced breast cancer. Breast Cancer Res Treat. 2010;122:181-188.
73. Bettini G, Blandamura S, Saia G, et al. Bevacizumab-related osteonecrosis of the mandible is a self-limiting disease process. BMJ Case Rep. 2012;2012. doi:10.1136/bcr-2012-007284.

74. Brunamonti Binello $P$, Bandelloni R, Labanca $M$, et al. Osteonecrosis of the jaws and bevacizumab therapy: a case report. Int J Immunopathol Pharmacol. 2012;25(3):789-791.

75. Tzermpos F, Ismail A, Pavli $M$, et al. Osteonecrosis of the mandible in a patient with lung adenocarcinoma undergoing anti-angiogenic therapy with bevacizumab. Oral Surg. 2015;9(1):40-46.

76. Fusco V, Fasciolo A, Pertino A, et al. Bevacizumab - related osteonecrosis of jaw in a rectal cancer patient never treated with bisphosphonates. Ann Stomatol. 2014. Available from: www.ncbi.nlm.nih. gov/pmc/articles/PMC4252873.

.- This is an early work documenting the onset of ONJ in patients receiving bevacizumab alone without the concomitant administration of agents that inhibit osteoclast activity.

77. Hopp RN, Pucci J, Santos-Silva AR, et al. Osteonecrosis after administration of intravitreous bevacizumab. J Oral Maxillofac Surg [Internet]. 2012 [cited 2011 Jul 14];70(3):632-635. doi:10.1016/j. joms.2011.02.104.

78. Pakosch D, Papadimas D, Munding J, et al. Osteonecrosis of the mandible due to anti-angiogenic agent, bevacizumab. Oral Maxillofac Surg. 2013;17(4):303-306. doi:10.1007/s10006-012-0379-9.

79. Santos-Silva AR, Belizário Rosa GA, Castro Júnior GD, et al. Osteonecrosis of the mandible associated with bevacizumab therapy. Oral Surg Oral Med Oral Pathol Oral Radiol. 2013;115:e32-e36.

80. Agostino NM, Gingrich R, Drabick JJ. Bevacizumab demonstrates prolonged disease stabilization in patients with heavily pretreated metastatic renal cell carcinoma: a case series and review of the literature. Adv Urol. 2010;2010:687043.

81. Magremanne M, Lahon M, De Ceulaer J, et al. Unusual bevacizumabrelated complication of an oral infection. J Oral Maxillofac Surg. 2013;71:53-55.

82. Ngamphaiboon N, Frustino $\mathrm{JL}$, Kossoff EB, et al. Osteonecrosis of the jaw: dental outcomes in metastatic breast cancer patients treated with bisphosphonates with/without bevacizumab. Clin Breast Cancer. 2011;11(4):252-257. doi:10.1016/j.clbc.2011.02.001.

83. Lescaille G, Coudert AE, Baaroun V, et al. Clinical study evaluating the effect of bevacizumab on the severity of zoledronic acidrelated osteonecrosis of the jawin cancer patients. Bone. 2014;58:103-107.

84. FDA report on aflibercept: Center for drug evaluation and research. Application number 1254180rig1s000. Clin Rev. Aflibercept/ Zaltrap. 100, 141. Available from: http://www.accessdata.fda.gov/ drugsatfda_docs/nda/2012/125418Orig1s000MedR.pdf

85. Van Cutsem E, Tabernero J, Lakomy R, et al. Addition of aflibercept to fluorouracil, leucovorin, and irinotecan improves survival in a phase III randomized trial in patients with metastatic colorectal cancer previously treated with an oxaliplatin-based regimen. J Clin Oncol. 2012; 30(28):3499-3506. [Epub 2012 Sep 4].

86. Tarhini AA, Frankel P, Margolin KA, et al. Aflibercept (VEGF Trap) in inoperable stage III or stage iv melanoma of cutaneous or uveal origin. Clin Cancer Res. 2011;17(20):6574-6581. doi:10.1158/10780432.CCR-11-1463.

87. Ponzetti A, Pinta F, Spadi R, et al. Jaw osteonecrosis associated with aflibercept, irinotecan and fluorouracil: attention to oral district. Tumori. 2015; doi:10.5301/tj.5000405. Epub ahead of print

88. Hartmann JT, Haap M, Kopp, HG et al. Tyrosine Kinase inhibitors- a review on pharmacology, metabolism and side effects. Curr Drug Metab. 2009;10(5):470-481.

89. Gilabert M, Provansal M, Cappiello M, et al. Buccodental side effects of sunitinib in patients with metastatic renal cell carcinoma. $\mathrm{Br} J$ Cancer [Internet]. 2013 [cited 2013 Sep 17];109(7):1750-1754. doi:10.1038/bjc.2013.516.

90. Balmor GR, Yarom N, Weitzen R. Drug-induced palate osteonecrosis following nasal surgery. Isr Med Assoc J. 2012;14:193-194.

91. Marino R, Orlandi F, Arecco F, et al. Osteonecrosis of the jaw in a patient receiving cabozantinib. Aust Dent J. 2014 Dec 4. doi:10.1111/adj.12254. [Epub ahead of print] 
92. Elisei R, Schlumberger MJ, Muller SP, et al. Cabozantinib in progressive medullary thyroid cancer. J Clin Oncol. 2013;31:3639-3646.

93. Bertoldo F, Silvestris F, Ibrahim T, et al. Targeting bone metastatic cancer: role of the mTOR pathway. Biochim Biophys Acta. 2014;1845(2):248-254. doi:10.1016/j.bbcan.2014.01.009.

94. Prati V, Ortega C, Aglietta M Caso clinico di Osteonecrosi da inibitore di m-TOR. Abstract Convegno Osteonecrosi dei mascellari (ONJ): ruolo della Rete Oncologica del Piemonte e della Valle d'Aosta Update 2011 (32). [2015 Jan 2]. Available from: http://www.reteoncologica.it/ images/stories/ONJ/alessandria\%20onj\%202011-abstract.pdf

95. Gnant M. The role of mammalian target of rapamycin (mTOR) inhibition in the treatment of advanced breast cancer. Curr Oncol Rep. 2013;15(1):14-23. doi:10.1007/s11912-012-0277-1.

96. Martini V, Bonacina R, Mariani U. Osteonecrosis of the jaw in a patient treated with zoledronic acid and everolimus: a case report. Ann Stomatol. 2014. Available from: www.ncbi.nlm.nih.gov/pmc/ articles/PMC4252873

97. Baselga J, Campone M, Piccart M, et al. Everolimus in postmenopausal hormone-receptor-positive advanced breast cancer. N Engl J Med. 2012;366(6):520-529. doi:10.1056/NEJMoa1109653.

98. Piccart M, Hortobagyi GN, Campone $M$, et al. Everolimus plus exemestane for hormone-receptor-positive, human epidermal growth factor receptor-2-negative advanced breast cancer: overall survival results from BOLERO-2†. Ann Oncol. 2014;25(12):23572362. doi:10.1093/annonc/mdu456.

99. Gnant M, Baselga J, Rugo HS, et al. Effect of everolimus on bone marker levels and progressive disease in bone in BOLERO-2. J Natl Cancer Inst. 2013;105(9):654-663. doi:10.1093/jnci/djt026.
100. FDA report on everolimus. Available from: http://www.accessdata. fda.gov/drugsatfda_docs/label/2015/022334s029lbl.pdf

101. Aapro M, Andre F, Blackwell K, et al. Adverse event management in patients with advanced cancer receiving oral everolimus: focus on breast cancer. Ann Oncol. 2014;25(4):763-773. doi:10.1093/annonc/ mdu021.

102. Owosho A, Scordo M, Yom SK, et al. Osteonecrosis of the jaw a new complication related to ipilimumab. Oral Oncology. 2015; doi:10.1016/j.oraloncology.2015.08.014. (Impact Factor: 3.61)

103. Edwards BJ, Gounder M, McKoy JM, et al. Pharmacovigilance and reporting oversight in US FDA fast-track process: bisphosphonates and osteonecrosis of the jaw. Lancet Oncol. 2008;9(12):1166-1172. doi:10.1016/S1470-2045(08)70305-X.

104. Fusco V Baraldi A, Fasciolo A, et al. ONJ: Impact of Italian patients, and role of Italian physicians, dentists, and researchers in the growing evidence of a "new" disease. Working Paper of Public Health (12/2012) (online journal). Available from: http://www.ospe dale.al.it/AllegatiPaginaDescrizione/Paper-12_2012_new.pdf.

.. This work documents how in Italy a large number of ONJ cases (several thousands) has been observed and Italian physicians and dentists had contributed largely to knowledge and awareness of the disease with conferences, papers, and publications.

105. Kruger TB, Sharikabad MN, Herlofson BB. Bisphosphonate-related osteonecrosis of the jaw in four Nordic countries and an indication of under-reporting. Acta Odontol Scand. 2013;71(6):1386-1390.

106. De Boissieu P, Trenque T. Standardized classification unsuitable for spontaneous reporting: the example of osteonecrosis of the jaw. Expert Opinion Drug Saf. 2015;14(7):1015.21. 